\title{
Compensatory Pelvic Retro-Rotation Associated with a Decreased Quality of Life in Patients with Normal Sagittal Balance
}

\author{
Tetsuya Shimokawa ${ }^{1,2}$, Kei Miyamoto ${ }^{3}$, Akira Hioki ${ }^{4}$, Takahiro Masuda ${ }^{5}$, \\ Kazunari Fushimi ${ }^{2}$, Hiroyasu Ogawa ${ }^{1}$, Kazuichiro Ohnishi ${ }^{1}$, Haruhiko Akiyama ${ }^{2}$ \\ ${ }^{I}$ Department of Orthopaedic Surgery, Ogaki Tokushukai Hospital, Gifu, Japan \\ ${ }^{2}$ Department of Orthopaedic Surgery, School of Medicine, Gifu University, Gifu, Japan \\ ${ }^{3}$ Department of Orthopaedic Surgery, Gifu Municipal Hospital, Gifu, Japan \\ ${ }^{4}$ Spine Center, Matsunami General Hospital, Gifu, Japan \\ ${ }^{5}$ Spine Center, Kizawa Memorial Hospital, Gifu, Japan
}

Study Design: Cross-sectional observational study.

Purpose: To examine whether pelvic rotation as a compensatory mechanism for sagittal imbalance is related to quality of life (QOL). Overview of Literature: Poor sagittal alignment is associated with compensatory pelvic retroversion and decreased QOL. Whether the compensatory pelvic tilt (PT) influences $00 \mathrm{~L}$ is unclear.

Methods: Overall, 134 subjects aged $\geq 20$ years with lower back pain were included (104 females; mean age, $70 \pm 9.8$ years). Sagittal vertical alignment (SVA) and PT were analyzed radiographically. Patients were stratified into three groups based on SVA values: good alignment (group G), intermediate alignment (group I), and poor sagittal alignment (group P). Patients in group I were further categorized into two groups: low PT and high PT. The Japanese Orthopaedic Association Back Pain Evaluation Questionnaire (JOABPEQ) was used for clinical assessment, and the scores were compared between groups.

Results: As SVA increased, PT and lumbar lordosis (LL) increased and decreased, respectively. PT and LL differed significantly between groups $\mathrm{G}$ and $\mathrm{P}$ ( $p<0.01$ for each comparison). Within group I, there was no significant difference in SVA between the high PT and low PT groups, suggesting that the high PT group had acquired a compensated sagittal balance. Importantly, all domains in the JOABPEQ (except for lower back pain) were significantly lower in the high PT group than in the low PT group $(p<0.05$ for every comparison).

Conclusions: This study showed that focusing solely on SVA as a single indicator can cause important losses in QOL to be overlooked in patients with lumbar disorders. Although pelvic retroversion can compensate for sagittal balance, it is associated with a significant decrease in QOL. To improve the assessment of patients with lumbar disorders, PT should be considered besides SVA.

Keywords: Sagittal alignment; Compensatory mechanism; Pelvic tilt; Low back pain; Japanese Orthopaedic Association Back Pain Evaluation Questionnaire

Received Aug 26, 2020; Revised Oct 25, 2020; Accepted Nov 5, 2020

Corresponding author: Kei Miyamoto

Department of Orthopaedic Surgery and Spine Center, Gifu Municipal Hospital, 7-1 Kashima Cho, Gifu City, Gifu 500-8513, Japan

Tel: +81-58-251-1101, Fax: +81-58-252-1335, E-mail: kei@bg8.so-net.ne.jp 


\section{Introduction}

Sagittal alignment of the spine and pelvis is closely related to the maintenance of a stable posture with minimum energy expenditure. As people age, the sagittal alignment and flexibility of the spine may be altered by various factors, including intervertebral disc degeneration, soft tissue degeneration, and traumatic vertebral body deformity $[1,2]$. The loss of lumbar lordosis (LL) and/or increase in pelvic tilt (PT) are typical signs of imbalanced sagittal alignment [3-5]. As sagittal imbalance can cause mechanical pain and neurological symptoms, mechanisms such as pelvic rotation, hip extension, and knee flexion are often gradually applied to compensate for spinal misalignment [6].

The use of such compensatory mechanisms can maintain posture during standing and walking, resulting in an unchanged sagittal vertical axis (SVA), an indicator of global sagittal alignment [7]. However, compensatory mechanisms eventually become insufficient because of the progression of the underlying pathology and longstanding excessive load, at which point the sagittal balance becomes abnormal $[1,2]$. This results in an increased SVA, which is associated with a decreased quality of life (QOL) $[8,9]$. However, in daily clinical practice, we encounter patients with back problems who have a normal SVA but an increased PT, raising the question of whether pelvic retroversion as a compensatory mechanism for sagittal imbalance could be associated with deterioration of QOL. However, information on compensated sagittal imbalance is scarce in the literature.

Thus, we conducted a study to elucidate the relationship between SVA and PT as an indicator of pelvic rotation and to investigate the association between PT and QOL within the context of lower back pain. We hypothesized that when SVA is compensated by PT, QOL is already declining.

\section{Materials and Methods}

\section{Patient selection}

This cross-sectional study included consecutive patients who visited the outpatient spine clinic of our hospital between April 2012 and September 2013 with the primary complaint of chronic lower back pain. The inclusion criteria were age $\geq 20$ years and the accurate completion of the Japanese Orthopaedic Association Back Pain Evaluation
Questionnaire (JOABPEQ). Exclusion criteria included lower extremity deformities and contracture, a history of spinal surgery, spinal tumors, acute spinal injury, neuromuscular diseases, rheumatoid arthritis, and collagen disease.

\section{Statement of ethics}

All patients provided written informed consent during their first visit. The institutional review board of Gifu University Hospital approved this study (approval no., 26-350).

\section{Radiographic assessment}

Sagittal radiographic parameters were calculated from whole spine sagittal radiographs obtained from patients in a freestanding posture while adopting the fists-on-clavicle position. SVA was defined as the horizontal distance from the posterior sacral plate corner to the vertebral body of C7. Pelvic incidence (PI) was measured as the angle between a line perpendicular to the sacral plate at its midpoint and a line connecting this point to the femoral head axis. PT was measured as the angle between the vertical line and a line joining the hip axis with the center of the sacral superior plate [10-12]. LL was measured from the superior endplate of L1 to the superior endplate of S1. To quantify the flattening of the lumbar spine in relation to pelvic morphology, the variable "PI minus LL" was calculated, and its contribution to QOL was assessed [9].

\section{Clinical assessment}

The JOABPEQ and Visual Analog Scale (VAS) scores were used to assess lower back pain, leg pain, and numbness. The JOABPEQ is a patient-based tool used to evaluate the QOL of patients with disorders characterized by lower back and/or leg pain [13-15]. It enables the specific evaluation of pain, gait, lumbar spine dysfunction, social activity impairment, and psychological impact. The score ranges from 0 to 100 points, with lower scores indicating a poorer QOL $[16,17]$.

\section{Definition and stratification of sagittal vertical axis and pelvic tilt}

Among asymptomatic Japanese adults, an SVA of -2.3 to $3.0 \mathrm{~cm}$ (mean, $3.2 \pm 4.7 \mathrm{~cm}$ ) is considered the standard spi- 


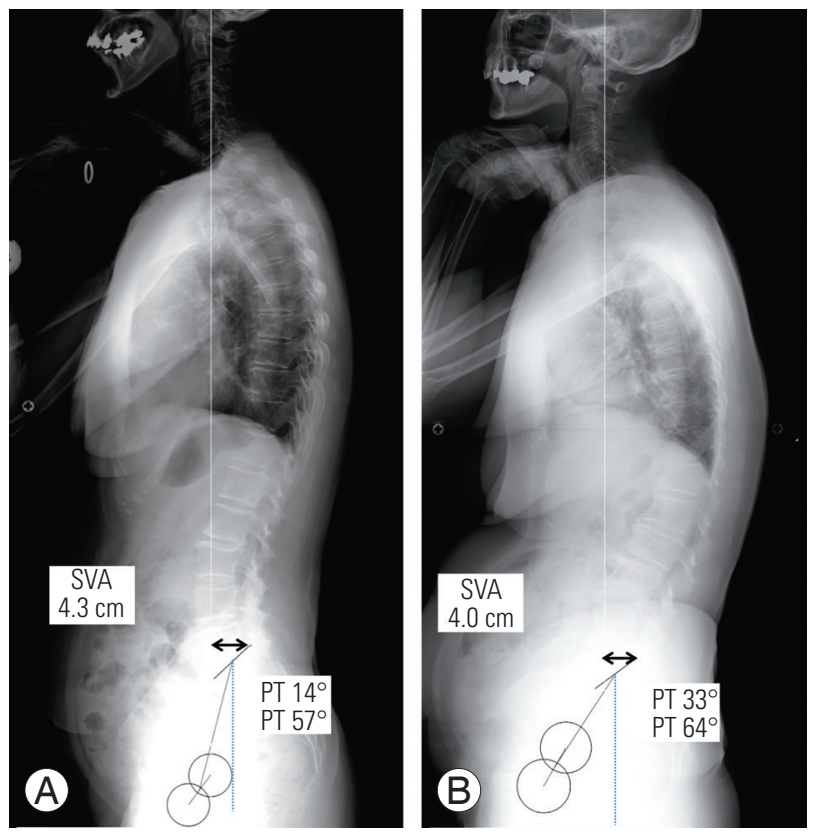

Fig. 1. Radiographs of representative cases in the low and the high pelvic tilt (PT) groups. (A) Low PT group: sagittal vertical alignment (SVA) of $43 \mathrm{~mm}$ and PT of $14^{\circ}$ are both within the normal ranges and the patient was classified into the low PT group. (B) High PT group: SVA of $40 \mathrm{~mm}$ is within the normal range but PT is $33^{\circ}$ and the patient was classified into the high PT group.

nal sagittal alignment, and intermediate spinal alignment is defined by an SVA of $4-9.5 \mathrm{~cm}$, according to the Scoliosis Research Society-Schwab adult spinal deformity classification $[9,18,19]$. Therefore, we stratified our patients into three groups based on SVA: good alignment (group G, SVA -3 to $3 \mathrm{~cm}$ ), intermediate alignment (group I, SVA $3.1-8 \mathrm{~cm}$ ), and poor alignment (group P, SVA $>8 \mathrm{~cm}$ ). Group I was further subdivided into a low PT group (no functioning pelvic compensatory mechanism, $\mathrm{PT} \leq 25^{\circ}$ ) (Fig. 1A) and a high PT group (presence of a functioning pelvic compensatory mechanism, $\mathrm{PT}>25^{\circ}$ ) (Fig. 1B).

\section{Statistical analyses}

Statistical analyses were conducted using Graph Pad Prism ver. 5.01 statistical software (GraphPad Software, La Jolla, CA, USA). The minimum sample size needed was 111 patients. Statistical comparisons of sagittal parameters and JOABPEQ scores in each group were made using the Kruskal-Wallis and Mann-Whitney $U$-tests for non-parametric analysis. Pearson's correlations were used to assess the correlation between PT and PI-LL and each domain of the JOABPEQ. The significance level was set at $p<0.05$ for all analyses.

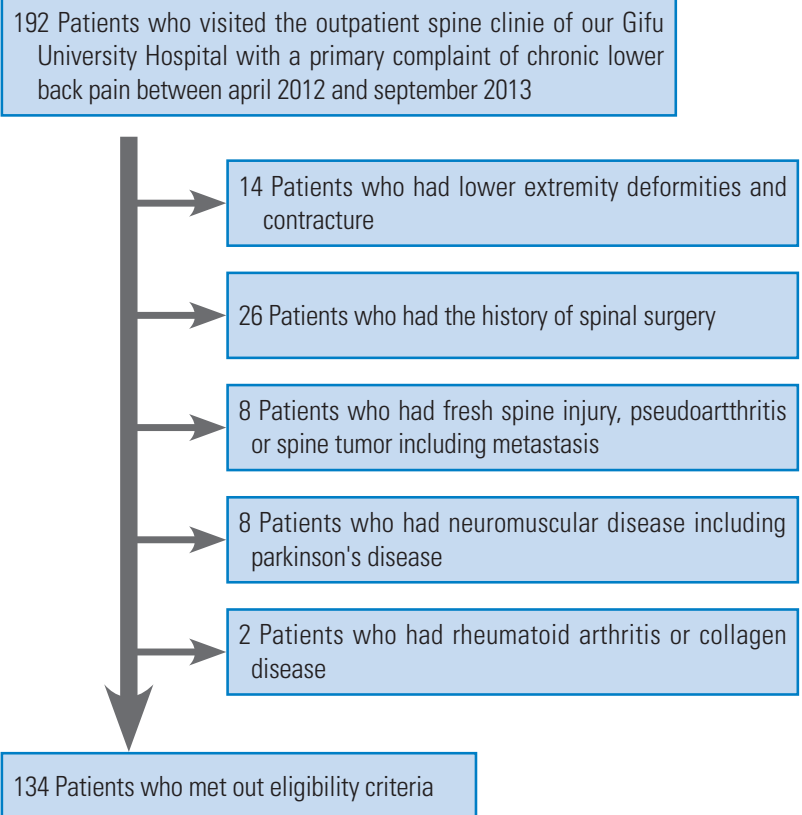

Fig. 2. Flowchart of patient enrollment.

\section{Results}

\section{Patient characteristics}

Based on the inclusion and exclusion criteria, 134 patients (104 females and 30 males) were included in this study (Fig. 2). Of these, 32 patients (23.9\%) were stratified into group G, 57 (42.5\%) into group I (29 [21.6\%] into the low PT and 28 [20.8\%] into the high PT group), and 45 (33.5\%) into group P. The overall mean age was $70 \pm 9.8$ years, and the mean ages in groups $\mathrm{G}$, low PT, high PT, and P were $67.6,69.0,70.4$, and 72.1 years, respectively. The male/female ratio was $9 / 23$ in group G, 7/22 in the low PT group, $3 / 25$ in the high PT group, and 11/34 in group P. There were no significant differences between groups in terms of age and male/female ratio.

\section{Sagittal radiographic parameters}

Fig. 3 shows the comparison of sagittal parameters between groups G, I, and P. In all groups, PT tended to increase along with increases in SVA. There were significant differences in PT between groups $\mathrm{G}$ and $\mathrm{P}$ ( $p<0.01$ for each comparison). Overall, LL decreased progressively as SVA increased. As PI did not change, PI-LL tended to increase accordingly. There were significant differences in LL and PI-LL between groups I and $\mathrm{P}$ ( $p<0.01$ for both comparisons). 


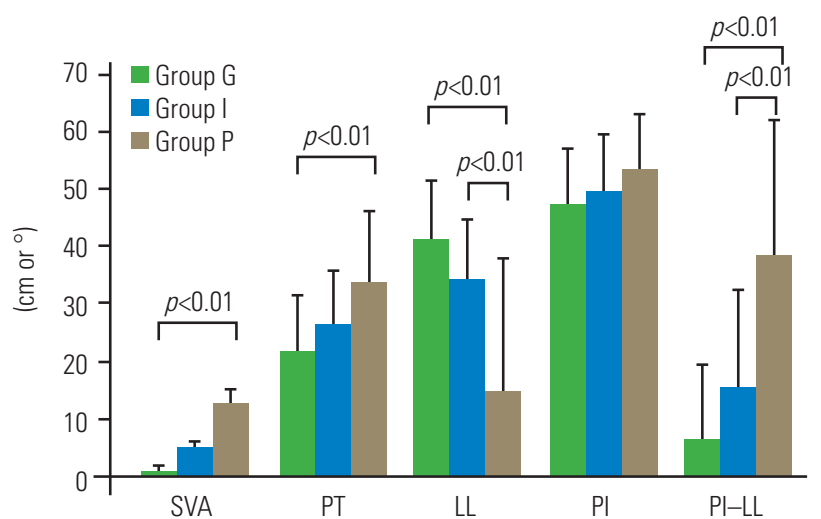

Fig. 3. Comparison of sagittal parameters between the three sagittal alignment groups: G (good), I (intermediate), and P (poor). SVA, sagittal vertical alignment; PT, pelvic tilt; LL, lumbar lordosis; PI, pelvic incidence; PI-LL, PI minus LL.

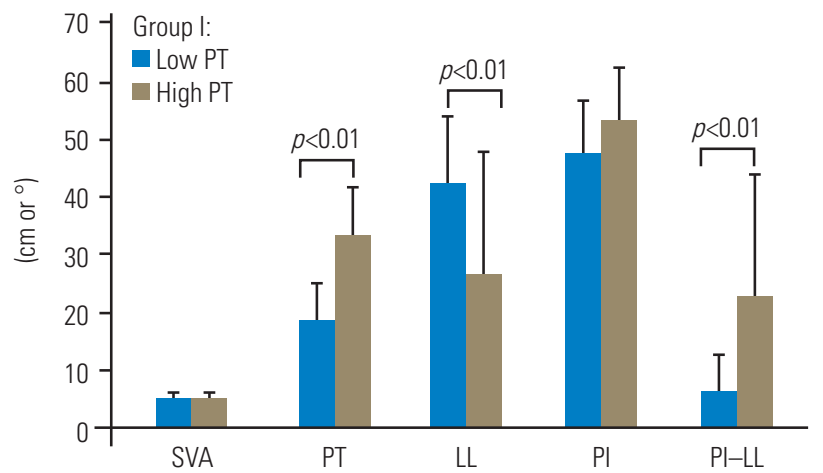

Fig. 4. Comparison of sagittal parameters between the low pelvic tilt (PT) and high PT groups. SVA, sagittal vertical alignment; LL, lumbar lordosis; PI, pelvic incidence; PI-LL, PI minus LL.

Fig. 4 shows the comparison between parameters in the low and high PT groups. Expectedly, PT was significantly higher in the high PT group than in the low PT group $(p<0.01)$, but no significant difference in SVA was seen between the two groups. LL was significantly lower in the high PT group than in the low PT group $(p<0.01)$, and PI-LL was significantly higher in the high PT group than in the low PT group $(p<0.01)$.

\section{Japanese Orthopaedic Association Back Pain Evalua- tion Questionnaire}

Groups G, I, and P were compared for each domain of the JOABPEQ (Fig. 5). The scores for lower back pain, lumbar function, and walking ability differed significantly between groups I and $\mathrm{P}(p<0.05,0.01$, and 0.01 , respectively) but not between groups $G$ and I. The social life function score differed significantly between groups $G$ and P $(p<0.01)$ but not between groups $\mathrm{G}$ and I or between

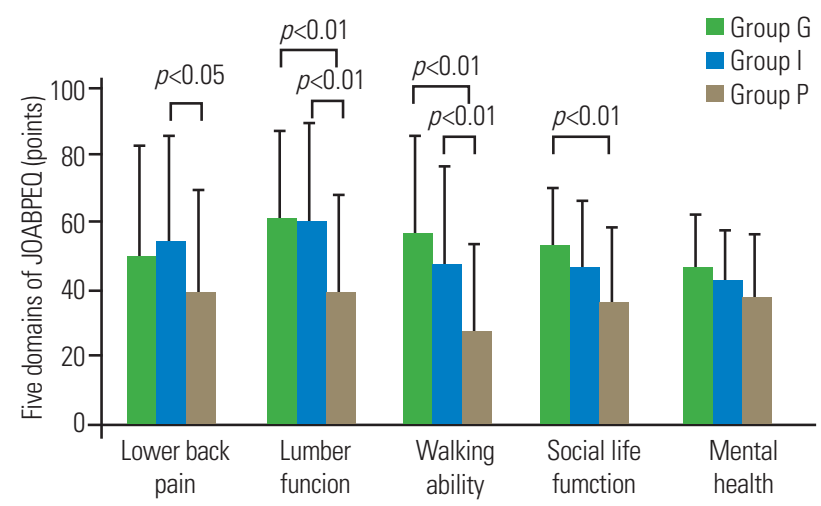

Fig. 5. Comparison of Japanese Orthopaedic Association Back Pain Evaluation Questionnaire (JOABPEO) scores between the three sagittal alignment groups: G (good), I (intermediate), and P (poor).

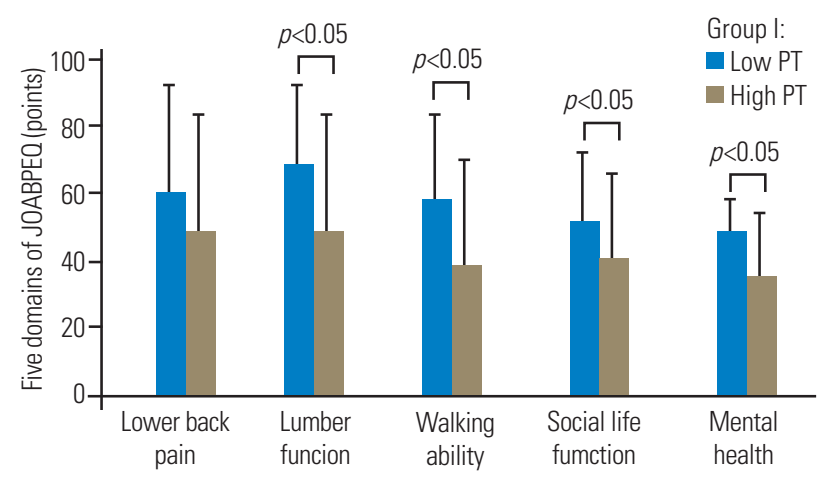

Fig. 6. Comparison of Japanese Orthopaedic Association Back Pain Evaluation Questionnaire (JOABPEQ) scores between the low pelvic tilt (PT) and high PT groups.

Table 1. Correlation between the pelvic tilt and the Japanese Orthopaedic Association Back Pain Evaluation Questionnaire domains

\begin{tabular}{lcc} 
Variable & Correlation coefficient $(r)$ & $p$-value \\
\hline Lower back pain & -0.15 & 0.261 \\
\hline Lumbar function & -0.33 & 0.009 \\
Walking ability & -0.23 & 0.09 \\
Social life function & -0.23 & 0.08 \\
\hline Mental health & -0.37 & 0.004 \\
\hline
\end{tabular}

groups I and P. There were no significant differences in the mental health score between groups.

The low and high PT groups were also compared for each domain of the JOABPEQ (Fig. 6). The scores for lumbar function, walking ability, social life function, and mental health were significantly lower in the high PT group than in the low PT group ( $p<0.05$ for each comparison).

A significant correlation was found between PT and three domains of the JOABPEQ: lumbar function, walk- 
ing ability, and mental health (Table 1).

\section{Visual Analog Scale scores for pain and numbness}

Analysis of VAS scores for lower back pain, leg pain, and numbness revealed no significant differences between groups $\mathrm{G}, \mathrm{I}$, and $\mathrm{P}$ or between the low and high PT groups (Fig. 7A, B).

\section{Discussion}

Several reports have suggested that increased PT is related to lower back pain and QOL $[12,18]$. However, when normal sagittal balance with compensatory pelvic retrorotation was compared with non-compensated normal sagittal balance, a difference in QOL was not discernible. The most important finding in the present study was that in patients with intermediate sagittal balance, JOABPEQ scores were significantly lower in patients with high PT than in patients with low PT. This suggests that the use of a compensatory mechanism (pelvic retroversion) to correct spinal imbalance is associated with a decline in QOL, even when the SVA remains unchanged.
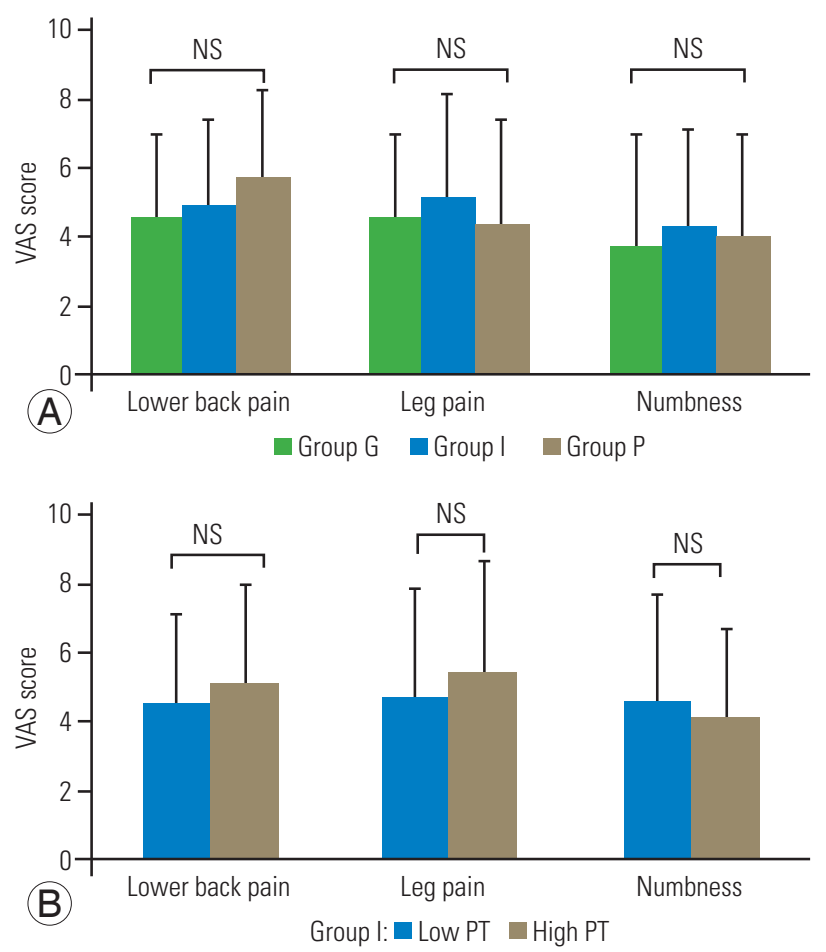

Fig. 7. Comparison of Visual Analog Scale (VAS) scores between (A) the three sagittal alignment groups $\mathrm{G}$ (good), I (intermediate), and P (poor) and P; (B) the low pelvic tilt (PT) and high PT groups. NS, not significant.
Pelvic rotation is considered to be an important mechanism to compensate for sagittal imbalance, up to the point at which physiological spinal alignment is lost $[12,20]$. Barrey et al. [2] proposed an algorithm to analyze a patient's global balance status by considering the presence of compensatory mechanisms in the spine (excessive cervical lordosis and lumbar retrospondylolisthesis), pelvis (retroversion), and lower limb (knee flexion and extension of the ankle joint). In the present study, PT increased as SVA increased and became clearly high when SVA was above the normal range (group P). This indicates that PT may be an important compensation mechanism and indicator of worsening SVA, appearing immediately before SVA deviates from the normal range.

Several previous reports suggest that pelvis-related parameters are important when analyzing the misalignment associated with spinal deformity $[6,21,22]$. Although SVA is directly linked to QOL [8], there is wide inter-patient variability $[9,18]$; therefore, pelvic-related parameters have emerged as accurate predictors of changes in QOL. Lafage et al. [23] reported that pelvic retroversion and anterior tilt of the trunk are associated with a decreased QOL, with PT and SVA being two important factors in this regard. However, until this study, little detail was known about the relationship between PT as a compensatory mechanism and QOL.

In the present study, there was a significant difference in LL (but not in SVA) between the high PT and low PT groups, implying that pelvic retroversion compensates for sagittal imbalance to maintain spinal alignment. Interestingly, QOL was significantly lower in the high PT group than in the low PT group, despite the comparable SVA. Moreover, the QOL of the low PT group was equivalent to that of patients with normal SVA (group G), whereas the QOL of the high PT group did not differ from that of the patients with high SVA (group P, detailed data not shown), suggesting that the onset of $\mathrm{PT}$ as a compensatory mechanism is accompanied by a decrease in QOL. Furthermore, PT was closely related to changes in some of the domains of the JOABPEQ score (Table 1). Taken together, these results strongly suggest that in cases of sagittal imbalance where spinal alignment is effectively maintained by PT, the QOL is adversely affected to a degree similar to cases with poor spinal alignment. Thus, PT is an important predictor of QOL decline, as inferred from its close correlation with PI-LL, which is considered as a good QOL indicator [18]. 
Kanemura et al. [24] reported that the JOA score for lower back pain did not significantly differ between patients with compensatory sagittal alignment and those with normal sagittal alignment. Similarly, in the present study, there were no significant differences between patients with compensatory alignment (the high PT group) and those with normal sagittal alignment (the low PT group) in VAS scores for lower back pain, leg pain, or numbness nor in lower back pain score in the JOABPEQ. However, scores in other domains such as lumbar function, walking ability, social life function, and mental health were significantly lower in the high PT group than in the low PT group. These results show that pelvic retroversion affects lumbar function and ability rather than painful symptoms, implying that this compensatory mechanism causes lumbar spine function deterioration. Presumably, the persistent muscle contraction of the spine and hip extensors required to maintain pelvic retroversion could produce general and local muscle fatigue. Further studies assessing the relationship between pelvic retroversion and decreases in QOL in patients with compensated sagittal balance may provide a clinical answer to this hypothesis.

There are several limitations to this study. First, asymptomatic volunteers were not included. Understanding the distribution of SVA and PT in asymptomatic individuals would help to understand the relationship between spinal alignment and PT. Second, in Japan, the prevalence of lower back pain is higher in women than in men, and the tendency is especially higher in individuals above 60 years old [25], so a higher ratio of women is expected. However, the male-to-female ratio was more biased toward women in the present study than in previous reports. Third, although lower extremity deformity and contracture were excluded in our study, occult lower extremity degenerative diseases such as very early osteoarthritis or degenerative meniscus rupture might have affected activities of daily living (ADL) and influenced the present result. Additionally, possible lumbar spinal stenosis in several patients might have affected walking ability and social life, thus biasing the present results. Fourth, some lumbar spinal canal stenosis cases, in which LL may be affected, were probably included among our study sample, which was designed to enroll patients with adult spinal deformity. Fifth, our classification based on SVA and PT was in accordance with the Scoliosis Research Society-Schwab adult spinal deformity classification and mean spinal alignment values in the Japanese population but remains to be validated.

\section{Conclusions}

In our study, we confirmed the primary hypothesis; although pelvic retroversion can compensate for sagittal balance, this mechanism is associated with impaired QOL and ADL. Focusing on SVA as a single parameter could lead to significant losses in QOL being overlooked in patients with lumbar disorders. Understanding the severity of lumbar degenerative disease and its compensatory mechanisms could help predict changes in QOL and thus determine the optimal timing for surgery. Therefore, we consider that including PT in addition to SVA as a routine parameter in assessing lumbar disorders is essential for a better knowledge of the clinical problem and should lead to improved patient management.

\section{Conflict of Interest}

No potential conflict of interest relevant to this article was reported.

\section{Author Contributions}

TS designed the study, collected, analysed and interpreted the data and drafted the article. KM conceived the idea, and revised the manuscript. Other authors revised the manuscript.

\section{References}

1. Ferrero E, Ould-Slimane M, Gille O, Guigui P; French Spine Society (SFCR). Sagittal spinopelvic alignment in 654 degenerative spondylolisthesis. Eur Spine J 2015;24:1219-27.

2. Barrey C, Roussouly P, Perrin G, Le Huec JC. Sagittal balance disorders in severe degenerative spine: can we identify the compensatory mechanisms? Eur Spine J 2011;20(Suppl 5):626-33.

3. Diebo BG, Varghese JJ, Lafage R, Schwab FJ, Lafage V. Sagittal alignment of the spine: what do you need to know? Clin Neurol Neurosurg 2015;139:295-301.

4. Redaelli A, Berjano P, Aebi M. Focal disorders of the spine with compensatory deformities: how to define them. Eur Spine J 2018;27(Suppl 1):59-69. 
5. Jeon I, Kim SW. Retrolisthesis as a compensatory mechanism in degenerative lumbar spine. J Korean Neurosurg Soc 2015;57:178-84.

6. Obeid I, Hauger O, Aunoble S, Bourghli A, Pellet N, Vital JM. Global analysis of sagittal spinal alignment in major deformities: correlation between lack of lumbar lordosis and flexion of the knee. Eur Spine J 2011;20(Suppl 5):681-5.

7. Dubousset J. Three-dimensional analysis of the scoliotic deformity. In: Weinstein SL, editor. Pediatric spine: principles and practice. New York (NY): Raven Press; 1994. p. 479-96.

8. Glassman SD, Bridwell K, Dimar JR, Horton W, Berven $S$, Schwab F. The impact of positive sagittal balance in adult spinal deformity. Spine (Phila Pa 1976) 2005;30:2024-9.

9. Schwab FJ, Blondel B, Bess S, et al. Radiographical spinopelvic parameters and disability in the setting of adult spinal deformity: a prospective multicenter analysis. Spine (Phila Pa 1976) 2013;38:E803-12.

10. Schwab F, Patel A, Ungar B, Farcy JP, Lafage V. Adult spinal deformity-postoperative standing imbalance: how much can you tolerate?: an overview of key parameters in assessing alignment and planning corrective surgery. Spine (Phila Pa 1976) 2010;35:2224-31.

11. Legaye J, Duval-Beaupere G, Hecquet J, Marty C. Pelvic incidence: a fundamental pelvic parameter for three-dimensional regulation of spinal sagittal curves. Eur Spine J 1998;7:99-103.

12. Jackson RP, Phipps T, Hales C, Surber J. Pelvic lordosis and alignment in spondylolisthesis. Spine (Phila Pa 1976) 2003;28:151-60.

13. Fukui M, Chiba K, Kawakami M, et al. Japanese Orthopaedic Association Back Pain Evaluation Questionnaire: part 2. Verification of its reliability: the Subcommittee on Low Back Pain and Cervical Myelopathy Evaluation of the Clinical Outcome Committee of the Japanese Orthopaedic Association. J Orthop Sci 2007;12:526-32.

14. Fukui M, Chiba K, Kawakami M, et al. Japanese Orthopaedic Association back pain evaluation questionnaire: part 3. Validity study and establishment of the measurement scale: Subcommittee on Low Back Pain and Cervical Myelopathy Evaluation of the Clinical Outcome Committee of the Japanese Orthopaedic Association, Japan. J Orthop Sci 2008;13:173-9.

15. Ohtori S, Ito T, Yamashita M, et al. Evaluation of low back pain using the Japanese Orthopaedic Association Back Pain Evaluation Questionnaire for lumbar spinal disease in a multicenter study: differences in scores based on age, sex, and type of disease. J Orthop Sci 2010;15:86-91.

16. Uesugi K, Sekiguchi M, Kikuchi S, et al. Lumbar spinal stenosis associated with peripheral arterial disease: a prospective multicenter observational study. J Orthop Sci 2012;17:673-81.

17. Hiyama A, Watanabe M, Katoh H, Sato M, Sakai D, Mochida J. Evaluation of quality of life and neuropathic pain in patients with low back pain using the Japanese Orthopedic Association Back Pain Evaluation Questionnaire. Eur Spine J 2015;24:503-12.

18. Schwab F, Ungar B, Blondel B, et al. Scoliosis Research Society-Schwab adult spinal deformity classification: a validation study. Spine (Phila Pa 1976) 2012;37:1077-82.

19. Kanemura T, Yoshida G, Ishikawa Y. Sagittal spinopelvic alignment in an asymptomatic Japanese population: comparison of Western population. J Spine Res 2011;2:52-8.

20. Pourtaheri S, Sharma A, Savage J, et al. Pelvic retroversion: a compensatory mechanism for lumbar stenosis. J Neurosurg Spine 2017;27:137-44.

21. Lamartina C, Berjano P, Petruzzi M, et al. Criteria to restore the sagittal balance in deformity and degenerative spondylolisthesis. Eur Spine J 2012;21(Suppl 1):S27-31.

22. McKay G, Torrie PA, Dempster G, Bertram W, Harding I. The relationship between sacral kyphosis and pelvic incidence. Asian Spine J 2018;12:74-9.

23. Lafage V, Schwab F, Patel A, Hawkinson N, Farcy JP. Pelvic tilt and truncal inclination: two key radiographic parameters in the setting of adults with spinal deformity. Spine (Phila Pa 1976) 2009;34:E599606.

24. Kanemura T, Satake K, Matsumoto A, Yamaguchi $\mathrm{H}$, Imakama S. Standing posture and low back pain: classification of the standing posture in the radiological spino-pelvic alignment. Spine Spinal Cord 2012;25:391-8.

25. Yoshimura N, Akune T, Fujiwara S, et al. Prevalence of knee pain, lumbar pain and its coexistence in Japanese men and women: the Longitudinal Cohorts of Motor System Organ (LOCOMO) study. J Bone Miner Metab 2014;32:524-32. 